\title{
Prospects for Measurements of the $\pi \rightarrow e v$ Branching Ratio
}

\section{Douglas Bryman}

University of British Columbia

Vancouver, Canada V6T2A3

E-mail: doug@triumf.ca

The $\pi \rightarrow e v / \pi \rightarrow \mu \nu$ branching ratio provides unique access to physics beyond the Standard Model due to the extraordinary precision of its SM prediction and the potential for highly accurate measurements. The motivation and prospects for new measurements presently underway at PSI and TRIUMF will be discussed. 


\section{Introduction}

Precise measurements of rare muon, pion, and $\mathrm{K}$ meson decays which have unambiguous Standard Model (SM) predictions enable exacting tests of current theories and searches for new physics at extremely high mass scales. Prominent among these processes, is the $\pi \rightarrow e v$ branching ratio which has been calculated to the highest precision for weak interactions involving hadrons.

The best test of the SM hypothesis of charged current universality comes from the decay ratio $R_{e / \mu}=\frac{\Gamma(\pi \rightarrow e v+\pi \rightarrow e v \gamma)}{\Gamma(\pi \rightarrow \mu \nu+\pi \rightarrow \mu v \gamma)}$ which has been measured to a precision of $0.4 \%$ in comparison with calculations that are an order of magnitude more precise. The most stringent test of e- $\mu$ universality $\left(g_{e} / g_{\mu}=0.9985 \pm 0.0016[1,2]\right)$ comes from measurements of $R_{e / \mu}$ followed closely by measurements of $\tau$ decay $\left(g_{e} / g_{\mu}=0.9999 \pm 0.0021\right.$ [3]). Marciano [4] pointed out that the branching ratio $R_{e / \mu}$ is sensitive to the longitudinal component of the W coupling (scalar and vector), while the $\tau$ decay tests the transverse component (vector).

In the $\mathrm{SM}$, electroweak radiative corrections to the $\pi \rightarrow l v, l=e, \mu$ decay rates are finite and calculable when expressed in terms of renormalized parameters. After including small structure dependent effects and the leading 2-loop logarithmic corrections one finds the SM prediction[4,5]: $\quad R_{e / \mu}^{S M}=1.2353(4) \times 10^{-4}$ where the error is very small but still quite conservative. This prediction is to be compared with the average $\mathrm{R}_{e / \mu}^{\exp \pi}=1.231(4) x 10^{-4}$ of experimental results from TRIUMF and PSI experiments which found $\mathrm{R}_{e / \mu}^{\exp \pi}=1.2265(34)(44) x 10^{-4}[1]$ and $\quad \mathrm{R}_{e / \mu}^{\exp \pi}=1.2346(35)(36) x 10^{-4}[2] . \quad$ The new experiments at PSI and TRIUMF aim to improve the precision of $\mathrm{R}_{e / \mu}^{\exp }$ by a factor of 5 or more, thereby confronting the SM prediction to better than $\pm 0.1 \%$. At that level, new physics effects could appear as a deviation from expectations or, in the absence of a deviation, strong new constraints on new physics hypotheses will be placed.

Candidate examples of the new physics probed by PIENU include heavy neutrino mixing [1] as well as high scale four fermion operators due to excited gauge bosons (e.g. from extra dimensions), and theories with leptoquarks, compositeness or charged Higgs bosons. Because of strong helicity-suppression in the SM $\pi \rightarrow e v$ decay, it is extremely sensitive to helicityunsuppressed couplings such as those due to pseudoscalar and scalar interactions. Since new pseudoscalar effects come in as interference terms with the dominant axial-vector term, such contributions are proportional to $1 / m_{H}^{2}$, where $m_{H}$ is the mass of a hypothetical heavy particle exchanged. This is in contrast to the $1 / m_{H}^{4}$ dependence in lepton flavor-violating decays like $\mu \rightarrow$ er .

Ignoring small contributions from $\pi \rightarrow \mu \nu$ decay, with the presence of pseudoscalar interactions, the deviation of the branching ratio from the SM prediction can be parameterized 
as $1-\frac{R_{e / \mu}^{N e w}}{R_{e / \mu}^{S M}} \sim \frac{\sqrt{2} \pi}{G_{\mu}} \frac{1}{\Lambda_{e P}{ }^{2}} \frac{m_{\pi}^{2}}{m_{e}\left(m_{d}+m_{u}\right)} \sim\left(\frac{1 T e V}{\Lambda_{e P}}\right)^{2} x 10^{3}$ where $\Lambda_{e P}$ is the mass scale of a new pseudoscalar interaction. This makes the measurement of the branching ratio at a $0.1 \%$ level sensitive to the mass scale of $1000 \mathrm{TeV}$ for pseudoscalar interactions. Scalar couplings arising from physics beyond the SM will also induce pseudoscalar interactions through loop corrections [6], and in most cases the $\pi \rightarrow e v$ branching ratio measurement provides substantially stronger limits than ones derived from super-allowed beta decay measurements and CKM unitarity. In the case of R-parity violating SUSY, the exchange of various generations of squarks may lead to a non-universal contribution that results in a comparable deviation, and the measurement of $R_{e / \mu}$ places substantial constraints on the possible size of R-parity violating effects[7]. In other approaches $R_{e / \mu}$ constrains theories involving sterile neutrinos (see for example [8]), and Majorons [9]. Several interesting new ideas (e.g. a hypothesis involving neutrino mass generated from the QCD quark condensate [10] and effects involving lepton flavor violation [11]) have been presented recently in which the $\pi \rightarrow e v$ branching ratio plays an important role in constraining new physics.

\section{New Experiments}

\subsection{The TRIUMF PIENU Experiment}

The new PIENU experiment is a refinement of the technique used in the previous TRIUMF experiment[1]. The branching ratio will be obtained from the ratio of positron yields from the $\pi \rightarrow e v$ decay and from the $\pi \rightarrow \mu \rightarrow e$ chain decay. By measuring positrons from the decays $\pi \rightarrow e v$ and $\pi \rightarrow \mu \rightarrow e$ in a non-magnetic spectrometer many normalization factors, such as the solid angle of positron detection, cancel to first order, and only small energydependent effects, such as those for multiple Coulomb scattering (MCS) and positron annihilation, need to be corrected for. Major improvements in precision stem from the use of a superior calorimeter, high speed digitizing of all pulses, Si strip tracking, and higher statistics.

Figure 1 shows the PIENU experimental setup, in which $\mathrm{B}$ and $\mathrm{T}$ indicate beam and telescope plastic scintillation counters, respectively. A $75 \mathrm{MeV} / \mathrm{c} \pi+$ beam from the TRIUMF M13 line will be identified by B1 and B2 scintillators, and stopped in a target consisting of an array of plastic scintillators principally, a 2-cm diameter, 1-cm thick stopping counter sandwiched by two 2-mm thick disc-shaped counters. Fine tracking near the target will be provided by two sets of single-sided $(\mathrm{X}, \mathrm{Y})$ silicon-strip counters located immediately upstream and downstream of the target assembly. In order to keep a low background level arising from 'old' muons in the target region and to minimize potential distortions in the time spectrum due to pile-up, the beam rate will be kept low. The telescope scintillation counters T1-3 covering the front side of the large Brookhaven National Laboratory NaI(Tl) crystal, BINA. BINA is a $48 \mathrm{~cm}$ diameter $\mathrm{x} 48$ $\mathrm{cm}$ long cylindrical single crystal instrument which has shown excellent energy resolution [12]. In the PIENU configuration energy resolution of $\Delta E / E \sim 2-3 \%(F W H M)$ at $66 \mathrm{MeV}$, a factor of 2 better than in the previous TRIUMF measurement [1] is anticipated. 


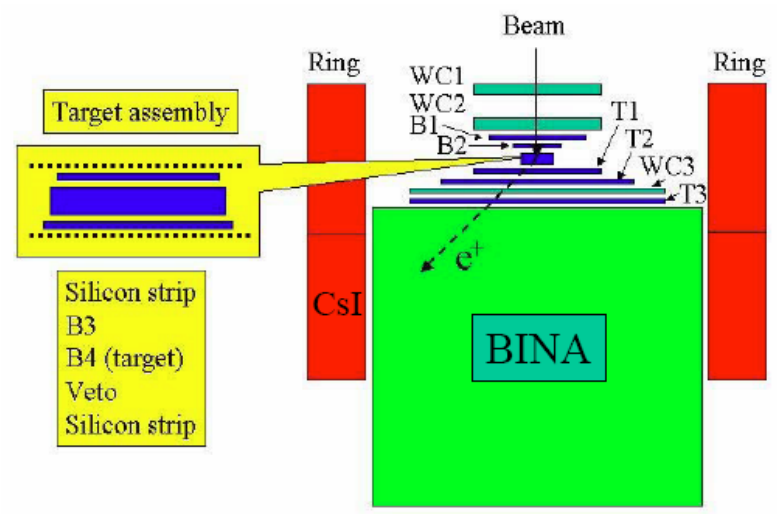

Figure 1. TRIUMF PIENU experiment setup.

A three-layer wire chamber (WC3) located next to the T3 counter provides position information of the positron for evaluation of shower leakage effects and correction of the path length in the $\mathrm{T}$ counters for $\mathrm{dE} / \mathrm{dx}$ measurements. The solid angle of the telescope is $25 \%$. The "Ring" in the Figure 1 is a $17-\mathrm{cm}$ thick cylinder, composed of $9725-\mathrm{cm}$ long pure CsI crystals used to capture shower leakage from BINA. Improvement in statistics will come from using a larger solid angle by an order of magnitude with a longer running period, and from greater precision of the tail correction measurement [1].

A total of $2 \times 10^{7} \pi \rightarrow e v$ events will be accumulated. With the detailed pulse shape information and reduced background due to pile-up compared to the previous experiment, an improvement factor of 30 or more is expected, resulting in a statistical uncertainty of $<0.05 \%$ in the branching ratio. The improvements lead to an expected precision on the branching ratio $<0.08$ $\%$, which corresponds to a $0.04 \%$ uncertainty in the ratio of the gauge boson-lepton coupling constants $g_{e} / g_{\mu}$.

\subsection{The PSI PEN Exeriment}

At PSI the $3 \pi$ sr PIBETA CsI calorimeter built for a determination of the $\pi^{+} \rightarrow \pi^{0} \mathrm{ev}$ branching ratio [13] will be used. The PIBETA detector system illustrated in Figure 2 is based on a large acceptance 240-module pure CsI electromagnetic shower calorimeter. The CsI crystals are 12 radiation lengths thick and have shown an energy resolution of $12.8 \%$ (FWHM) at $66 \mathrm{MeV}$. The detector includes an active degrader $\mathrm{AD}$, a segmented active target AT, a 20bar cylindrical plastic scintillator veto PV for particle identification, a pair of tracking cylindrical multi-wire proportional chambers MWPC1/2 and an active cosmic veto shield. The beam counters and target region will be modified for the PEN experiment and pulses will be digitized at $500 \mathrm{MHz}$. 
(i)

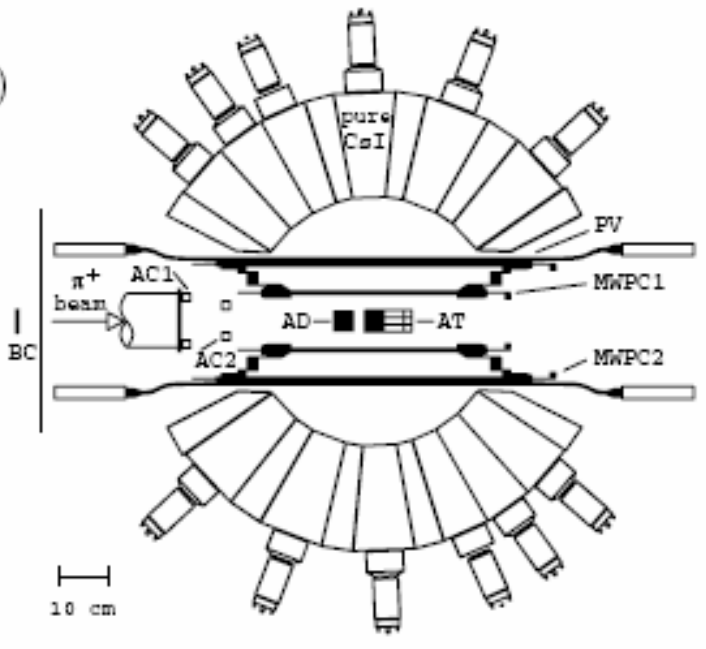

(ii)

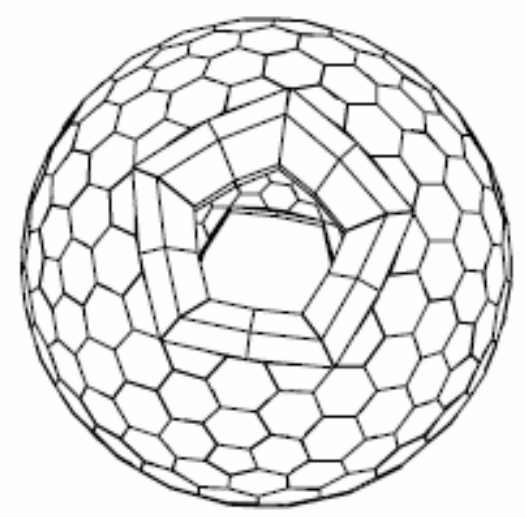

Figure 2. PIBETA detector: (i) cross-sectional view showing the major detector sub-systems, and (ii) 240-module pure CsI calorimeter geometry [14 ].

The PEN technique will be similar to that employed in the previous PSI experiment which used a nearly $4 \pi$ sr BGO spectrometer [2]. Large samples of $\pi \rightarrow e v$ decays have been recorded parasitically in the past which were used as normalization for $\pi^{+} \rightarrow \pi^{0} \mathrm{eV}$ with an accuracy of $<0.3 \%$. The setup was also used for the accurate studies of the radiative decays $\pi \rightarrow e v \gamma$ [15] and $\mu \rightarrow e v \overline{v \gamma}$ [14]. Based on this experience an improvement in precision for $R_{e / \mu}$ by almost an order of magnitude is expected.

\section{Summary}

Two new experiments PIENU and PEN measuring the $\pi \rightarrow e v$ branching ratio $R_{e / \mu}=\frac{\Gamma(\pi \rightarrow e v+\pi \rightarrow e v \gamma)}{\Gamma(\pi \rightarrow \mu \nu+\pi \rightarrow \mu v \gamma)}$ are in progress at TRIUMF and PSI aiming to improve the precision by an order of magnitude to $<0.1 \%$. These measurements will provide ever more stringent tests of the hypothesis of lepton universality and will be sensitive to indirect effects of new physics at extremely high mass scales $\mathrm{O}(1000 \mathrm{TeV})$. 


\section{References}

[1] D.I. Britton et al., Phys. Rev. Lett. 68, 3000 (1992); D.I. Britton et al., Phys. Rev. D46, R885 (1992); see also D.I. Britton et al., Phys. Rev. Lett. D46,885 (1992).

[2] G. Czapek et al., Phys. Rev. Lett. 70, 17 (1993).

[3] Particle Data Group, Review of Particle Physics, Phys. Lett. B592 (2004).

[4] W.J. Marciano and A. Sirlin, Phys. Rev. Lett. 71, 3629 (1993).

[5] M. Finkemeier, Phys. Lett. B387, 391 (1996); S.M. Berman, Phys. Rev. Lett. 1, 468 (1958); T. Kinoshita, Phys. Rev. Lett. 2, 477 (1959); M.V. Terentev, Yad. Fiz. 18, 870 (1973); and W. Marciano and T. Krupovnickas, private communication.

[6] B.A. Campbell and D.W. Maybury, Nucl. Phys. B709, 419 (2005).

[7] M. J. Ramsey-Musolf, Phys. Rev. D62, 056009 (2000).

[8]_J. Maalampi, V. Sipilainen, and I. Vilja, Phys. Rev. D67,113005 (2003).

[9] A. Smirnov, and R. Funchal, Phys .Rev. D74,013001 (2006).

[10] H. Davoudiasl and L. Everett, Phys. Lett. B634, 55 (2006).

[11] A. Masiero, P. Paradisi, and R. Petronzio, hep-ph/0511289; A. Masiero, P. Paradisi hep-ph/0609262 (2006).

[12] G. Blanpied et al., Phys. Rev. Lett. 76, 1023 (1996).

[13] D. Pocanic, E. Frlez, V.A. Baranov et al., Phys. Rev. Lett. 93, 181803-1 (2004).

[14] E. Frlez, Nuclear Physics B (Proc. Suppl.) 162, 148 (2006).

[15] E. Frlez et al., Phys. Rev. Lett.93, 181804 (2004). 